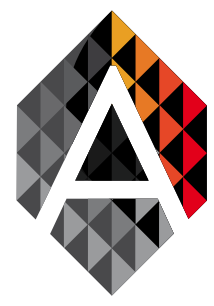

ADCAIJ: Advances in Distributed Computing and Artificial Intelligence Journal Regular Issue, Vol. 8 N. 4 (2019), 33-40

eISSN: $2255-2863$

DOI: http://dx.doi.org/10.14201/ADCAIJ2019843340

\title{
Multi-agent system for anomaly detection in Industry 4.0 using Machine Learning techniques
}

\author{
Nuria Mateos García \\ Faculty of Chemical Sciences, University of Salamanca, Plaza de los Caídos, 1, Salamanca, 37008, Spain \\ nurimateos@usal.es
}

$\begin{array}{ll}\text { KEYWORD } & \text { ABSTRACT } \\ \begin{array}{l}\text { Industry 4.0; } \\ \begin{array}{l}\text { Multiagent } \\ \text { system; Machine } \\ \text { Learning; Failure } \\ \text { diagnosis }\end{array} \\ \text { nectivity and globalization. The interrelation between different areas has penetrated } \\ \text { the indunt of information is available in plants and is growing increasingly, also aided } \\ \text { by the network computing services offered by cloud computing or edge computing. } \\ \text { That is why it's necessary to carry out complex fusion methods and data analysis using } \\ \text { Machine Learning techniques to address specific industrial requirements and needs. } \\ \text { The central challenge of industry 4.0 from the perspective of data science is to predict } \\ \text { the history within monitored processes, providing as much information as possible, } \\ \text { avoiding them and stave off severe economic losses. This article will show a review } \\ \text { of the application of Artificial Intelligence (AI) techniques such as Machine Learning } \\ \text { (ML) immersed in multi-agent systems (MAS) in Industry 4.0. For this, a bibliographic } \\ \text { search has been carried out in databases recognized as Science Direct, Google Schol- } \\ \text { ar, Scopus or Springer, filtering the investigations from 2018 to actuality. The article } \\ \text { concludes pointing out possible future lines and the importance of transition towards } \\ \text { the implementation of new technologies for the competitiveness of factories. }\end{array} \\ \end{array}$

\section{Introduction}

The industry has suffered a big revolution with the new paradigm of Industry 4.0. The fourth industrial revolution seeks to change the industry in an intelligent environment. This change is driven by the adoption of new information and communication technologies applied to industrial processes and products.

Numerous tasks are performed in a factory, each with own objectives. This article incorporates the term intelligent agent, which is an independent software entity that receives and manages the inputs of the environment. In the industry there are many machines equipped with all kinds of sensors that measure their own data, such as modifications or disturbances and measure the data of its environment. Agent uses this information to control the behavior of the machines through cooperation with other agents. The agent's intention is to serve as a central coordinator capable of communicating with other software-based solutions and controlling the existing machinery through known communication protocols (Carvalho et al., 2019).

Nuria Mateos García

Multi-agent system for anomaly detection in Industry 4.0 using Machine Learning techniques
ADCAIJ: Advances in Distributed Computing and Artificial Intelligence Journal Regular Issue, Vol. 8 N. 4 (2019), 33-40 elSSN: 2255-2863 - http://adcaij.usal.es Ediciones Universidad de Salamanca - CC BY NC DC 
Internet of Things (IoT) techniques are used to retrieve information from sensors and send it securely to a centralized server. From the perspective of data science, thanks to the development of IoT technology it's possible to collect data from industrial processes in real time. IoT includes the use of devices such as smart helmets, drones, robots, smart glasses, smart pipes for real-time assistance, security to detect possible damage and send all that data in real time to control them (Hanga and Kovalchuk, 2019) and provides real-time information to manufacturers since a component can fail at any time.

All this, coupled with the increasing availability of Internet connection, with the future launch of $5 \mathrm{G}$ networks in the smart grid paradigm, will allow communication between agents, machines and industrial applications. Communication is critical in control systems, and even more when there are multiple machines.

The cloud solution is the center of the proposed architecture by providing data storage, public web hosting,central modeling optimization and configuration data distribution and parameterization on all edge nodes. One of the key tasks performed by the solution in the cloud is to merge several models of edge devices, optimize them and send the optimal version back to the edge to update remote devices (Carvalho et al., 2019). Edge Computing allows data produced by IoT devices to be processed locally, closer to where it's acquired instead of sending it to data centers or Clouds, requiring less ongoing bandwidth and storage cost and takes advantage of distributed computing strategies and reduces latency and the amount of information traversing the network. Beyond the convenience of Edge Computing in terms of communication resources, this computation paradigm has important implications not only on the selection of the hardware platform for the use case at hand, but also in the modeling stage. Data fusion and analysis are implemented locally, so radically new model design strategies must be devised and developed to distribute, share and incrementally learn from the prognostic knowledge gained from locally captured data. Beyond the convenience of Edge Computing in terms of communication resources, this computation paradigm has important implications not only on the selection of the hardware platform for the use case at hand, but also data fusion and analysis are implemented locally (Diez-Olivan et al., 2019).

With the fourth industrial revolution, or smart industry, thanks to the improvements and incorporation of devices (IoT), there has been an epoch of great amount of data (Big Data). This paradigm shift allows extracting relevant knowledge of the monitored assets by adopting intelligent monitoring and data fusion strategies (DiezOlivan et al., 2019). Datasets collected from Big Data sensors and external sources, such as benchmarks with other machines, market data or external factors are very complex and traditional data processing applications cannot handle them and a set is required of techniques and technologies for massive and complex data (Wolfert et al., 2017). To avoid this and achieve prediction, machine learning techniques are used.

Both machine learning and multi-agent systems serve to make decisions, although at different scales. Multiagent systems are responsible for automating and coordinating complex and distributed tasks, communication and collaboration of agents, facilitating supervision and management. Machine learning techniques are applied to make predictions and detect anomalies. The sum of the ability of MAS to solve complex and unpredictable problems with the potential of ML allows reaching key solutions in the applications of industry 4.0 and are integrated into MAS as independent entities intended to analyze incoming data, to avoid agent learning. In addition, when modeling agents it's impossible to predict all the situations that the agent can experience, much less in dynamic environments, so it isn't a practical task to previously define the ideal behavior of an agent.

The ideal paradigm is multi-agent learning, which consists of an environment of multiple agents capable of learning, adapting and adjusting their behavior to the environment and interacting between agents (Hernandez-Leal et al., 2019). Machine learning techniques will be incorporated into multi-agent systems, so it will consist of assistant data collection agents and another central agent that integrates machine learning algorithms to detect abnormal behaviors. If an anomaly is detected, the central agent must identify it and notify the corresponding agents to generate a new decision to adjust the behavior of physical assets. Through the utilities of Internet services, it's also possible to graphically show the knowledge acquired to operators, so that they can carry out a maintenance program, avoiding critical stops and large costs in factories.

The objective of this work is to investigate multi-agent systems and how they can facilitate the prediction of anomalies in Industry 4.0 through the different machine learning techniques.

Nuria Mateos García

Multi-agent system for anomaly detection in Industry 4.0

using Machine Learning techniques
ADCAIJ: Advances in Distributed Computing and Artificial Intelligence Journal Regular Issue, Vol. 8 N. 4 (2019), 33-40 eISSN: 2255-2863 - http://adcaij.usal.es Ediciones Universidad de Salamanca - CC BY NC DC 
The article is structured as follows: Section 2 reviews the operation of the multi-agent systems; Section 3 reviews how anomalies can be detected in Industry 4.0; Section 4 reviews the different Machine Learning techniques; finally, Section 5 contain the final conclusions on the state of the art of the subject discussed.

\section{Multi-agent system}

To handle the large amount of information provided by the multiple assets of the factories, it's an intelligent solution to establish a multi-agent system composed of multiple intelligent agents interacting with each other to solve difficult and impossible problems to solve individually.

The structure of this system could consist of several agents, each of which is modeled as a virtual digital shell of each asset in the production line and collects the data generated by the asset, and a central agent (middle-ware running on the server) to which it's implemented a learning algorithm to identify abnormal behavior. The different tasks that the multi-agent system could perform are the following:

- Cleaning agents and data monitoring: each agent processes and monitors the data generated by each asset in the production process.

- Data Collection: each agent collects and stores the data and data characteristics of the assets to build the behavior model.

- Low level agents: these mobile agents form the first level of identification. They travel to each of their associated data cleaning agents, collect recent information and the extracted data feed the formulas, statistical methods that will classify the data and detect abnormal behavior. Agents send the data sample to the central agent running on the server. These agents collaborate to establish their level of suspicion to determine cooperatively if a suspicious action is more interesting in the presence of another suspicious activity.

- High-level agents: Maintain the data warehouse by combining knowledge and data of low-level agents. High-level agents apply data mining algorithms to discover associations and patterns. The central agent applies a statistical test or machine learning algorithm to detect if there is any abnormal behavior in the data.

- Group decision-making: if an anomaly is detected, the central agent must quickly identify the cause of the anomaly and notify the corresponding sub-agents to make the necessary decisions to adjust the behavior of physical assets.

- Interface agent: directs the operation and adjusts the behavior of the agents in the system, maintains the status reported by the mobile agents and provides access to the characteristic data store. For example, the central agent can send an order to shut down the machine to the agent that is involved in an anomaly.

\section{Detection of anomalies in Industry 4.0}

Manufacturing has been evolving to be increasingly intelligent and is data driven. In the era of industrial IoT, an intelligent production unit can be perceived as a large connected industrial system of parts, tools, materials, machines, inventory and logistics that can transmit data and communicate between all parties (Lade et al., 2017).

The current industry (immersed in the fourth industrial revolution), has very complex processes that entail the need to use new techniques of blurred and neuro-fuzzy control. For the analysis of data in manufacturing, a traditional data mining analysis or analyzing data with few characteristics is no longer enough, but the data obtained are many and very complex, since they are taken in real time. Recent advances in Big Data are used to transform this network data into a valid format for storage in a specific database, providing high capacity and fast query execution.

Nuria Mateos García

Multi-agent system for anomaly detection in Industry 4.0 using Machine Learning techniques
ADCAIJ: Advances in Distributed Computing and Artificial Intelligence Journal Regular Issue, Vol. 8 N. 4 (2019), 33-40 eISSN: 2255-2863 - http://adcaij.usal.es Ediciones Universidad de Salamanca - CC BY NC DC 
Statistical tools are used to analyze and summarize data, which are then used by Machine Learning tools to predict what will happen in the future.

Through the services Internet utilities, this knowledge is shown graphically to operators to carry out an informed maintenance program. Therefore, the tasks to be performed for anomaly detection are:

1. Collect, analyze and process massive data (in real time) and controlled by intelligent agents.

2. Apply machine learning techniques in the multi-agent system to predict the state of machinery, what we call predictive model.

The objective is to integrate ML into the MAS to detect anomalies in a supervised way. For this, the architecture can be client-server style (CS). The anomaly detection algorithm helps the central agent distinguish abnormal behavior from production activities. The algorithm is based on:

- Feature selection: it's essential to provide meaningful information to the machine to learn. Deep learning architectures may not need that information, because they can create them. But this would imply having a large set of data that will also need a large amount of computing power.

- Fit parameters: these are parameters that algorithms use to control different process properties, such as the size of the model, the maximum number of iterations in the learning data or the type of regularization.

- Probability: calculate the probability of a value given other values and see if it's lower or higher than the predefined limits as threshold, since it can be a significant characteristic.

\subsection{Massive data and data collection}

IoT collects real-time information from sensors and processes and sends them to the cloud, which allows to deal with industrial problems in real time. A certain number of data captured from multiple sensors with the process conditions (working hours, asset loading, failure rates) and external conditions (temperature, humidity) are required. Massive data involves techniques such as Big Data where machine learning techniques are applied to make predictions. Given the large amount of data and process information exchange, it's appropriate to implement a multi-agent architecture for collaborative learning.

The way to capture and manage that data is explained in the Section 1 and 2. Statistical techniques are used to analyze and summarize the data, then they are used machine learning techniques, which imply in their future behavior. For example, histograms and correlation tables can be used to understand the relationship between data distribution and variables.

\subsection{Types of maintenance}

According to (Diez-Olivan et al., 2019), the different machine learning techniques to apply to vary depending on the purpose.

\subsubsection{Predictive maintenance}

Predictive maintenance consists in monitoring the state of the machinery to decide when a repair or replacement is needed to be given the levels of degradation of the parts, which avoids spending time in controlling the machinery when it isn't necessary and avoiding failures and stops. This has a very important application in modern industries and is possible thanks to the emerging technologies mentioned above: CPS, IoT, Big Data, IoS.

The objective is to predict the moment in which a failure will occur in a monitored equipment and know its severity in the production chain. Predictive models are based on a dataset of fault events that have occurred in the past, to which a learning algorithm is applied that discovers patterns that correlate the captured data of the monitored asset with an objective variable that characterizes the type of failure to be predicted (for example, probability of failure, severity of failure).

In the work proposed by (Shafiee and Sørensen, 2019) models or strategies are identified to optimize maintenance decisions and inspection procedures for various wind energy assets. In another work (Liu et al., 2019) 
is considered the health status and dummy age subjected to machine degradation. Another interesting case is the research conducted by (Ceruti et al., 2019) who introduce augmented reality as a visible tool for performing maintenance and spare parts (in aviation).

The different algorithms that can be applied to make these predictions are shown in the next section (Section 4).

\subsubsection{Descriptive maintenance}

Once the failure has occurred, uses data mining to provide information about the past and see what has happened and how it can be resolved and that the impact is minimal.

\subsubsection{Prescriptive forecast}

Sets the optimal actions as a result of a plant fault alarm. When the alarm is activated by a predictive model before the failure occurs, the actions of the prescriptive forecast models modify the working parameters and the variables of the industrial process eventually affected by the failure to reduce the chances of occurrence. If the alarm is triggered by a confirmed fault, these models are used to minimize their impact on industry production. It's often modeled as an optimization problem, whose objective(s) are often driven by the outcome of predictive forecasting models. Therefore, optimization solvers prevail within this category (Diez-Olivan et al., 2019).

Many times, hybridization of different types of models is required for multiple prognoses. For example, combining the predictive forecast to predict the probability that a machine will suffer a failure, with the prescriptive forecast, to adjust the machine configuration to less likely failures (Diez-Olivan et al., 2019).

\section{Machine Learning techniques}

When reviewing the literature, there are several examples of predictive models implanted to determine the state of the machinery, most come from changes in artificial intelligence or machine learning.

For the data analysis, Artificial Neural Network (ANNs) and Support Vector Machines (SVMs) can be applied, both to optimize the operations of the assets and for maintenance purposes. The operation with them is as follows: from the training samples, the invariant character vectors that are used as desired references for the classification of faults associated with the nearest neighbor classifier are extracted and then a single class support vector machine (SC-SVM) is used for the fault detection as show the investigations of (Hasan et al., 2019) and (Ma et al., 2018). SVM is very effective to detect and predict with signals collected in a short time and with high precision (Han et al., 2012).

(Martins et al., 2015) propose a machine learning technique in a multi-agent framework for online outliers' detection in Wireless Sensor Networks using least squares support vector machine along with a learning algorithm based on sliding windows. K Nearest Neighbor (KNN) algorithm was also used by (Zheng et al., 2019) for a semi-supervised Fisher discriminant analysis model for classification of industrial process failures. O'Mahony discovered that deep neural networks (DL) have demonstrated great success in the domain of large data (Chen et al., 2019) (Wolfert et al., 2017).

Projection methods (kernel methods, linear, non-linear and orthogonal projections to latent structures) (DiezOlivan et al., 2019) for the time-series dimensional reduction with adjustment of specific hyper parameters in Bayesian and regression can help the process feature extraction and make a more accurate prediction. Bayesian search is an excellent approach for optimizing hyper parameters of models applied to fault diagnosis as show the investigations of (Cabrera et al., 2020), (Pang et al., 2019) and (Ruiz-Sarmiento et al., 2020) obtained good results with a predictive model using the Naive Bayes algorithm for the maintenance of industrial machinery in the context of industry 4.0.

In systems composed of several complex processes it can be very difficult to carry out fault detection and diagnosis. It may be useful to use the Fuzzy C-means clustering technique combined with the wavelet transformation applied for the detection and diagnosis of operational failures of an industrial installation to separate the most volatile component that is the methylcyclohexane contained in the mixture liquid. For noise reduction in order to accurately determine the mapping of data to different kinds of faults in the Fuzzy Cmeans cluster

Nuria Mateos García

Multi-agent system for anomaly detection in Industry 4.0

using Machine Learning techniques
ADCAIJ: Advances in Distributed Computing and Artificial Intelligence Journal Regular Issue, Vol. 8 N. 4 (2019), 33-40 elSSN: 2255-2863 - http://adcaij.usal.es Ediciones Universidad de Salamanca - CC BY NC DC 
distillation column in combination with the wavelet transformation for noise reduction in order to accurately determine the data mapping to different kinds of distillation column failures, giving better results than with the exclusive use of Fuzzy C-means (Azzaoui et al., 2019). These types of methodologies based on fuzzy logic are almost not integrated in the industrial sectors, either because companies doubt their benefits or there are inconveniences of integration in terms of cost and time. However, it may be that new research demonstrates its applicability and that companies increasingly adopt them (Diez-Olivan et al., 2019).

(Peres et al., 2018) proposes a generic framework for data analysis and real-time supervision for Industry 4.0 predictive manufacturing systems deployed in a 4-node cluster. The unsupervised algorithms in problems with high-dimensional data using clustering techniques (Han et al., 2012), (Luo et al., 2019), (Zhang et al., 2019) and outlier detection methods (Ramchandran and Sangaiah, 2018) are also effective. (Tsang and Kwong, 2006) propose a multi-agent intrusion detection system in industrial network using ant colony clustering approach and unsupervised feature extraction.

(Paolanti et al., 2018) proposes random forest for predictive maintenance in Industry 4.0.

\section{Conclusions}

Based on the review of the bibliography, several conclusions can be drawn about the state of the art of multiagent systems that use machine learning techniques to detect anomalies in smart industry. First, that Industry 4.0 and intelligent manufacturing optimize manufacturing systems, reduce manufacturing costs, reduce the development cycle of new products, avoid failures and can count on fully integrated and automated production processes, with machines capable of self-administration and maintenance, which provide globally accessible information in real time through the Internet and different devices, facilitating the creation of cooperation networks with multi-agent architecture.

Another important aspect to consider is that to achieve this it's necessary to train people with the skills required by these new technologies (data management and analysis, online simulation, computer-assisted production, programming or predictive maintenance).

In this article, literature was reviewed on multi-agent systems for the detection of anomalies in Industry 4.0 using machine learning techniques. The different machine learning algorithms for the prediction of anomalies within the Industry 4.0 paradigm have been discussed.

In conclusion, the potential of ML for the detection of anomalies and the capabilities of MAS for the coordination and resolution of complex problems answer the research question and implementation in the smart factory context.

In conclusion, the potential of ML for the detection of anomalies and the capabilities of MAS for the coordination and resolution of complex problems answer the research question and implementation in the smart factory context.

\section{References}

Azzaoui, H., Mansouri, I., and Elkihel, B., 2019. Methylcyclohexane Continuous Distillation Column Fault Detection Using Stationary Wavelet Transform and Fuzzy C-means. Materials Today: Proceedings, 13:597606. ISSN 2214-7853. doi:https://doi.org/10.1016/j.matpr.2019.04.018. International Conference on Materials and Environmental Science, ICMES2018, Mohammed Premier University, Oujda, Morocco, April 26-28, 2018.

Cabrera, D., Guamán, A., Zhang, S., Cerrada, M., Sánchez, R.-V., Cevallos, J., Long, J., and Li, C., 2020. Bayesian approach and time series dimensionality reduction to LSTM-based model-building for fault diagnosis of a reciprocating compressor. Neurocomputing, 380:51-66. ISSN 0925-2312. doi:https: //doi. org/10.1016/j.neucom.2019.11.006.

Carvalho, A., Mahony, N. O., Krpalkova, L., Campbell, S., Walsh, J., and Doody, P., 2019. At the Edge of Industry 4.0. Procedia Computer Science, 155:276-281. ISSN 1877-0509. doi:https://doi.org/10.1016/j.procs.

Nuria Mateos García

Multi-agent system for anomaly detection in Industry 4.0

using Machine Learning techniques
ADCAIJ: Advances in Distributed Computing and Artificial Intelligence Journal Regular Issue, Vol. 8 N. 4 (2019), 33-40 elSSN: 2255-2863 - http://adcaij.usal.es Ediciones Universidad de Salamanca - CC BY NC DC 
2019.08.039. The 16th International Conference on Mobile Systems and Pervasive Computing (MobiSPC 2019), The 14th International Conference on Future Networks and Communications (FNC-2019), The 9th International Conference on Sustainable Energy Information Technology.

Ceruti, A., Marzocca, P., Liverani, A., and Bil, C., 2019. Maintenance in aeronautics in an Industry 4.0 context: The role of Augmented Reality and Additive Manufacturing. Journal of Computational Design and Engineering, 6(4):516-526. ISSN 2288-4300. doi:https://doi.org/10.1016/j.jcde.2019.02.001.

Chen, H., Jiang, B., Zhang, T., and Lu, N., 2019. Data-driven and deep learning-based detection and diagnosis of incipient faults with application to electrical traction systems. Neurocomputing. ISSN 0925-2312. doi:https://doi.org/10.1016/j.neucom.2018.07.103.

Diez-Olivan, A., Ser, J. D., Galar, D., and Sierra, B., 2019. Data fusion and machine learning for industrial prognosis: Trends and perspectives towards Industry 4.0. Information Fusion, 50:92-111.

Han, J., Kamber, M., and Pei, J., 2012. 3 - Data Preprocessing. In Data Mining (Third Edition), The Morgan Kaufmann Series in Data Management Systems, pages 83-124. Morgan Kaufmann, Boston, third edition edition. ISBN 978-0-12-381479-1. doi:https://doi.org/10.1016/B978-0-12-381479-1.00003-4.

Hanga, K. M. and Kovalchuk, Y., 2019. Machine learning and multi-agent systems in oil and gas industry applications: A survey. Computer Science Review, 34:100191. ISSN 1574-0137. doi:https://doi.org/10. 1016/j. cosrev.2019.08.002.

Hasan, M. J., Islam, M. M., and Kim, J.-M., 2019. Acoustic spectral imaging and transfer learning for reliable bearing fault diagnosis under variable speed conditions. Measurement, 138:620-631. ISSN 0263-2241. doi:https://doi.org/10.1016/j.measurement.2019.02.075.

Hernandez-Leal, P., Kartal, B., and Taylor, M. E., 2019. A survey and critique of multiagent deep reinforcement learning. Autonomous Agents and Multi-Agent Systems, 33:750-797.

Lade, P., Ghosh, R., and Srinivasan, S., 2017. Manufacturing Analytics and Industrial Internet of Things. IEEE Intelligent Systems, 32:74-79.

Liu, Q., Dong, M., Chen, F., Lv, W., and Ye, C., 2019. Single-machine-based joint optimization of predictive maintenance planning and production scheduling. Robotics and Computer-Integrated Manufacturing, 55:173-182. ISSN 0736-5845. doi:https://doi.org/10.1016/j.rcim.2018.09.007. Extended Papers Selected from FAIM2016.

Luo, X., Fong, K., Sun, Y., and Leung, M., 2019. Development of clustering-based sensor fault detection and diagnosis strategy for chilled water system. Energy and Buildings, 186:17-36. ISSN 0378-7788. doi:https:// doi.org/10.1016/j.enbuild.2019.01.006.

Ma, S., Cheng, B., Shang, Z., and Liu, G., 2018. Scattering transform and LSPTSVM based fault diagnosis of rotating machinery. Mechanical Systems and Signal Processing, 104:155-170. ISSN 0888-3270. doi:https:// doi.org/10.1016/j.ymssp.2017.10.026.

Martins, H., Januario, F., Brito Palma, L., Cardoso, A., and Gil, P., 2015. A machine learning technique in a multi-agent framework for online outliers detection in Wireless Sensor Networks. pages 000688-000693. doi:10.1109/IECON.2015.7392180.

Pang, S., Yang, X., Zhang, X., and Lin, X., 2019. Fault diagnosis of rotating machinery with ensemble kernel extreme learning machine based on fused multi-domain features. ISA Transactions. ISSN 0019-0578. doi:https://doi.org/10.1016/j.isatra.2019.08.053.

Paolanti, M., Romeo, L., Felicetti, A., Mancini, A., Frontoni, E., and Loncarski, J., 2018. Machine Learning approach for Predictive Maintenance in Industry 4.0. pages 1-6. doi:10.1109/MESA.2018.8449150.

Peres, R. S., Rocha, A. D., Leitao, P., and Barata, J., 2018. IDARTS-Towards intelligent data analysis and real-time supervision for industry 4.0. Computers in Industry, 101:138-146. ISSN 0166-3615. doi:https://doi. org/10.1016/j.compind.2018.07.004.

Ramchandran, A. and Sangaiah, A. K., 2018. Chapter 11 - Unsupervised Anomaly Detection for High Dimensional Data - an Exploratory Analysis. In Sangaiah, A. K., Sheng, M., and Zhang, Z., editors, Computational Intelligence for Multimedia Big Data on the Cloud with Engineering Applications, Intelligent Data-Centric Systems, pages 233-251. Academic Press. ISBN 978-0-12-813314-9. doi: https://doi.org/10.1016/ B978-0-12-813314-9.00011-6.

Nuria Mateos García

Multi-agent system for anomaly detection in Industry 4.0 using Machine Learning techniques
ADCAIJ: Advances in Distributed Computing and Artificial Intelligence Journal Regular Issue, Vol. 8 N. 4 (2019), 33-40 elSSN: 2255-2863 - http://adcaij.usal.es Ediciones Universidad de Salamanca - CC BY NC DC 
Ruiz-Sarmiento, J.-R., Monroy, J., Moreno, F.-A., Galindo, C., Bonelo, J.-M., and Gonzalez-Jimenez, J., 2020. A predictive model for the maintenance of industrial machinery in the context of industry 4.0. Engineering Applications of Artificial Intelligence, 87:103289. ISSN 0952-1976. doi:https://doi.org/10.1016/j.engappai. 2019.103289.

Shafiee, M. and Sørensen, J. D., 2019. Maintenance optimization and inspection planning of wind energy assets: Models, methods and strategies. Reliability Engineering and System Safety, 192:105993. ISSN 09518320. doi:https://doi.org/10.1016/j.ress.2017.10.025. Complex Systems RAMS Optimization: Methods and Applications.

Tsang, C.-H. and Kwong, S., 2006. Multi-agent intrusion detection system in industrial network using ant colony clustering approach and unsupervised feature extraction. pages 51-56. ISBN 0-7803-9484-4. doi:10.1109/ ICIT.2005.1600609.

Wolfert, S., Ge, L., Verdouw, C., and Bogaardt, M.-J., 2017. Big Data in Smart Farming-A review. Agricultural Systems, 153:69-80. ISSN 0308-521X. doi:https://doi.org/10.1016/j.agsy.2017.01.023.

Zhang, H., Chen, H., Guo, Y., Wang, J., Li, G., and Shen, L., 2019. Sensor fault detection and diagnosis for a water source heat pump air-conditioning system based on PCA and preprocessed by combined clustering. Applied Thermal Engineering, 160:114098. ISSN 1359-4311. doi:https://doi.org/10.1016/j.applthermaleng. 2019.114098.

Zheng, J., Wang, H., Song, Z., and Ge, Z., 2019. Ensemble semi-supervised Fisher discriminant analysis model for fault classification in industrial processes. ISA Transactions, 92:109-117. ISSN 0019-0578. doi:https:// doi.org/10.1016/j.isatra.2019.02.021. 